\title{
Pratiques
}

Linguistique, littérature, didactique

$169-170 \mid 2016$

Enseignement/apprentissage de la langue, des textes et des discours. 40\&nbspans de Pratiques

\section{Comment puiser dans le répertoire langagier de l'apprenant lors de l'enseignement/apprentissage de la deuxième langue étrangère ?}

How to draw on the Learner's Language Repertoire when Teaching/Learning the Second Foreign Language?

Radosław Kucharczyk

\section{(2) OpenEdition}

Journals

Édition électronique

URL : http://journals.openedition.org/pratiques/3000

DOI : 10.4000 /pratiques.3000

ISSN : 2425-2042

Éditeur

Centre de recherche sur les médiations (CREM)

Référence électronique

Radosław Kucharczyk, " Comment puiser dans le répertoire langagier de l'apprenant lors de

l'enseignement/apprentissage de la deuxième langue étrangère ? », Pratiques [En ligne],

169-170 | 2016, mis en ligne le 30 juin 2016, consulté le 20 avril 2019. URL : http://

journals.openedition.org/pratiques/3000; DOI : 10.4000/pratiques.3000

Ce document a été généré automatiquement le 20 avril 2019.

(C) Tous droits réservés 


\section{Comment puiser dans le répertoire langagier de l'apprenant lors de l'enseignement/apprentissage de la deuxième langue étrangère?}

How to draw on the Learner's Language Repertoire when Teaching/Learning the Second Foreign Language?

Radosław Kucharczyk

\section{Introduction}

1 L'idée du plurilinguisme est omniprésente dans les documents européens concernant la politique éducative. Il va donc de soi qu'elle doit être aussi présente en classe de langue étrangère qui est un espace privilégiée pour le développement de la compétence plurilingue et qui constitue un objectif de prime importance dans le processus d'enseignement/apprentissage des langues vivantes. Dans le texte qui suit, nous caractériserons brièvement la compétence plurilingue, puis nous montrerons ses implications pratiques. L'article sera clos par la présentation des résultats de la recherche menée auprès d'élèves polonais âgés d'entre 15 et 16 ans apprenant le français en tant que LE2 (après l'anglais). L'objectif de cette recherche était d'examiner à quel point les élèves sont capables de puiser dans leur répertoire langagier pour rendre l'apprentissage du français plus efficace.

\section{Le plurilinguisme : un défi didactique mis en relief}

2 Si le bi/plurilinguisme constitue, depuis un bon moment, un objet de recherches en sociolinguistique, il commence à s'ancrer dans la pensée didactique, aussi bien sur le plan 
théorique que sur celui des pratiques de classe. En effet, déjà à partir des années 80 , « la visée de construction européenne et le développement de la circulation des personnes que celle-ci suppose ont en effet amené les experts du Conseil de l'Europe à promouvoir un renforcement de l'apprentissage des langues, non seulement à l'école, mais également tout au long de la vie hors du cadre scolaire» (Huver \& Springer 2011, p. 77). On peut donc constater que la voie vers le plurilinguisme est désormais ouverte, d'autant plus que le souci du renforcement des compétences langagières (y compris les compétences en langue maternelle) apparait dans plusieurs documents européens relatifs à l'éducation et à la formation. Citons à ce titre des documents tels la Recommandation du Parlement européen et du Conseil sur les compétences clés pour l'éducation (2006), la Stratégie européenne en faveur du multilinguisme (2008) ou encore le Cadre stratégique pour la coopération européenne dans le domaine de l'éducation et de la formation (2009). Il n'est donc pas étonnant de voir que les langues vivantes étrangères sont désormais valorisées dans les établissements scolaires. Comme le remarquent les concepteurs du Guide pour l'élaboration des politiques linguistiques éducatives en Europe (2003), cette tendance va de pair avec «l'accroissement de la demande sociale en langues, laquelle dépend, à son tour, de la prise de conscience plus diffusée que la maîtrise des langues étrangères fait désormais partie des compétences professionnelles» (Beacco \& Byram, 2003, p. 23). Ainsi, on consacre plus d'importance à l'enseignement des langues au cours de la scolarité (plus d'heures de cours, enseignement précoce: préélémentaire et élémentaire) tandis que l'offre « linguistique » dans les écoles devient de plus en plus variée. Ce point de vue est repris, voire renforcé dans les travaux ultérieurs du Conseil de l'Europe qui mènent vers l'élaboration d'un référentiel commun pour tous les pays membres, à savoir un Cadre européen commun de référence (désormais CECR - 2001) qui met en relief l'idée du plurilinguisme en proposant une nouvelle approche, dite plurilingue, qui suppose un travail constant sur le répertoire langagier de l'apprenant. En effet, cette approche interroge le mode traditionnel d'enseignement/apprentissage des langues où ces dernières sont traitées en tant qu'unités séparées, indépendantes les unes des autres. Au contraire, elle suppose un travail constant sur le répertoire langagier de l'apprenant «dans lequel toutes les capacités linguistiques trouvent leur place » (CECR, p. 11). Pour ce faire, l'individu puise dans son expérience linguistique, variable en fonction de son vécu langagier et culturel. Il n'est donc pas étonnant de constater que l'objectif majeur du processus d'enseignement/apprentissage des langues est désormais de développer la compétence plurilingue qui, à côté de la compétence interculturelle, garantirait le succès lors de la situation de communication exolingue. Or, la compétence plurilingue est définie en tant que "compétence à communiquer langagièrement [...] d'un acteur social qui possède, à des degrés divers, la maitrise de plusieurs langues [...]. On considèrera qu'il n'y a pas là superposition ou juxtaposition de compétences distinctes, mais bien existence d'une compétence complexe, voire composite, dans laquelle l'utilisateur peut puiser » ( $C E C R, p .129)$. Ainsi définie, la compétence plurilingue suppose le rejet de la dichotomie langue maternelle/langue(s) étrangère(s): l'individu plurilingue ne possède pas des compétences communicatives distinctes et séparées les unes des autres dans les langues qu'il maitrise, mais d'une seule compétence qui englobe tout son répertoire langagier dont il active différentes composantes en fonction du besoin communicatif. Il semble donc qu'une nouvelle perspective soit nécessaire dans le champ de la didactique des langues étrangères. Non seulement toute connaissance d'une langue est partielle, mais aussi toute connaissance, dans l'acceptation large du terme, est partielle dans le sens qu'elle n'est jamais parfaitement équilibrée entre ses composantes (par exemple, oral/ 
écrit ou compréhension/réception). Cela signifie que le modèle du locuteur natif - celui qui maitrise sa langue maternelle par excellence - est rejeté. Ce point de vue semble juste, car

les locuteurs natifs sont faits, et non nés, mais vous incluez en fait plusieurs niveaux différents de dialectes, d'éducation, de conscience, etc. dans ce concept global. Simplement pour donner ce qui pourrait s'avérer être un angle utile ou deux. Il y a des personnes étant super conscientes de la langue et de son usage - les poètes, les écrivains, les faiseurs de bons mots, les gens qui créent de nouveaux mots soit pour s'amuser, soit parce qu'ils en vivent ; et il y a des personnes qui sont, de façon générale, inconscientes de l'usage de leur langue ou de celle d'autres personnes (même si elles peuvent être capables de caractériser le discours d'une autre personne comme étant celui d'un «bouseux » voire même de l'imiter, sans pour autant pouvoir nommer ou identifier les éléments menant à cette caractérisation). (Paikeday, 2003, p. 33, notre traduction) ${ }^{1}$

3 Il résulte de ce qui précède que la compétence plurilingue est une compétence d'acquisition (Beacco \& Byram, 2003, p. 37), car chacun peut acquérir la maitrise de différentes langues et/ou variations linguistiques dans le contexte de formation ou hors ce contexte, parce que la capacité à apprendre des langues est tout à fait naturelle et accessible à tous. Ainsi, «le plurilingue n'est pas un locuteur d'exception, comme le polyglotte, et le plurilinguisme ne peut pas être considéré comme le privilège d'une élite de locuteurs "surdoués" »(ibid.). Néanmoins, il faut prendre en considération le fait que, même si le plurilinguisme est ordinaire, l'acquisition des langues est différente en fonction de la distance entre la L1 et la LE que l'individu apprend. Nous reviendrons à cette question dans la suite du présent article. Pour cette raison, la compétence dont dispose l'individu plurilingue n'est pas équilibrée (elle est donc partielle), car elle résulte de son répertoire qui n'est pas forcément homogène parce qu'il subit des modifications suite à l'expérience de l'individu. C'est pourquoi la compétence plurilingue est une compétence évolutive, car elle se greffe sur la bibliographie linguistique de l'individu (Robert \& Rosen, 2010, p. 56). Il est tout de même à noter que l'apprentissage d'une langue n'est pas impossible après la période de première enfance. Comme le remarquent J.-C. Beacco \& M. Byram (2003, p. 38), «si l'acquisition des langues s'effectue de manière sans doute spécifique dans la première enfance, cela ne signifie pas qu'ensuite, dans l'éducation primaire et au-delà, l'enrichissement des répertoires plurilingues soit impossible ou nécessairement plus difficile. Cela est souvent une question de besoin et de motivation ». Il faut aussi ajouter que la compétence plurilingue est perçue en tant que répertoire de ressources communicatives que l'interlocuteur active en fonction de ses besoins, d'autant plus que la situation de communication donnée n'est pas forcément gérée dans une seule langue ou une seule variété linguistique. Pour cette raison, la compétence plurilingue peut être décrite en tant que compétence fonctionnelle car elle permet à l'individu de se débrouiller lors de la situation de communication qui se déroule, au moins partiellement, dans une autre langue que sa langue maternelle. Il résulte de ce qui précède que le processus d'enseignement/apprentissage des langues devrait subir des modifications profondes. Il n'est plus question de travailler les langues de façon cloisonnée, mais il serait recommandé de puiser dans la synergie qui devrait se mettre en place entre les langues qui forment le répertoire plurilingue de l'individu. Pour ce faire, il faut développer chez les apprenants la conscience métalinguistique, condition sine qua non, de la compétence plurilingue, en leur proposant une activité dite réflexive sur le système linguistique en tant que tel, sans oublier de prendre en considération le contexte 
(non seulement social mais aussi affectif) dans lequel l'enseignement/apprentissage a lieu. C'est à ces éléments que nous allons consacrer le passage suivant.

\section{La compétence plurilingue : quelles implications didactiques?}

\subsection{Conscience métalinguistique}

Le travail sur la compétence plurilingue de l'apprenant en classe de langue nécessite une réflexion consciente sur le répertoire langagier dont il dispose. En effet, comme l'écrit Z. Chłopek (2011, p.98-100), la conscience métalinguistique englobe la capacité à expliquer différents aspects du code linguistique à l'aide du métalangage (il s'agit donc de la capacité acquise lors de la scolarisation), mais aussi le savoir que l'apprenant possède sur la langue. On peut également faire la distinction entre la conscience métalinguistique et la conscience interlinguistique ou, en d'autres termes, la conscience concernant les ressemblances et les différences entre différentes langues, tout en soulignant qu'il existe une interaction bien forte entre les deux types de conscience. Quant aux dimensions de la conscience relative au fonctionnement de la/des langue/s, on distingue l'analyse et le contrôle. L'analyse du savoir linguistique a un impact sur le développement des représentations mentales de la langue, tandis que le contrôle du savoir assimilé permet de recourir à des informations pertinentes lors d'une situation de communication donnée. Aussi bien l'analyse que le contrôle ont un impact sur le changement des représentations langagières et, par voie de conséquence, amorcent le développement de la compétence plurilingue par le fait que de nouvelles connexions interlinguales se mettent en place. Il découle de ce qui précède que la conscience métalinguistique est la capacité à :

- réfléchir sur la langue de façon non seulement abstraite mais aussi flexible ;

- reconnaitre un certain nombre de régularités (au niveau de la structure, de la morphologie, de la phonétique, etc.) ;

- percevoir et analyser la forme linguistique hors du contexte ;

- contrôler et manipuler la forme linguistique lors de la situation de communication (dans le contexte).

5 En classe de langue, il faudrait donc encourager les apprenants à la réflexion sur la langue et la communication lors des activités dites de conscientisation. Au niveau de la langue, cette activité englobe non seulement la réalisation des activités langagières (réception, production, interaction, de même que la médiation) mais aussi le cadre de la situation de communication. Comme le remarque J. Arditty (2008, p. 36), il s'agit d'une « sorte de veille permanente, d'attention flottante mais prête à se mobiliser, sur la conformité de leurs propres productions à leurs objectifs communicatifs aussi bien qu'aux diverses normes associées à la situation d'interlocution ». La tâche de l'enseignant est donc de faire émerger cette conscience auprès de ses apprenants ou, en d'autres termes, de passer des activités épilinguistiques (non conscientes) vers les activités métalinguistiques (conscientes). Ces activités seront ensuite automatisées par les élèves qui évalueront, de manière plus ou moins consciente, le contexte dans lequel l'activité linguistique en question se produit, afin de pouvoir pleinement profiter de toutes les composantes de leur répertoire linguistique. En effet, ce contrôle ou monitoring du contexte est une condition nécessaire à la réussite lors de l'activité linguistique car « il y a là un ensemble 
de domaines qui entrent en interaction dans la compétence langagière et qui sont des objets potentiels de la conscience langagière pour tout sujet parlant, quelles que soient l'ampleur et la diversité de son registre linguistique » (ibid.). Il faut aussi souligner que l'activité réflexive ne concerne pas seulement le travail sur les compétences linguistiques, mais qu'elle devrait également englober différents systèmes de langues, tels le lexique, la grammaire ou la phonologie (Bourguignon \& Candelier, 2014, p. 91). De plus, la réflexion devrait être inscrite de façon naturelle dans le processus d'apprentissage/enseignement, car « apprendre, c'est faire des hypothèses à partir à la fois du connu et de ce que l'on perçoit du nouveau, afin, soit d'intégrer ce nouveau dans le connu, soit d'aménager le connu pour qu'il puisse intégrer le nouveau» (ibid., p. 92). Perçu de cette façon, l'apprentissage est traité en tant que processus actif : il y a un va-et-vient constant entre le savoir déjà possédé par l'apprenant et le savoir qu'il est en train d'acquérir. C'est ainsi que se construit le nouveau savoir qui débouchera, par la suite, sur la construction de nouveaux savoir-faire. Adapter un tel point de vue rend le travail sur le répertoire langagier de l'apprenant possible et efficace, et permet, par suite du processus de conscientisation, de développer le répertoire langagier de l'apprenant, ce qui a un impact positif sur le développement de sa compétence plurilingue.

\subsection{Attitudes envers les langues}

6 Éveiller la conscience métalinguistique chez les apprenants en classe de langue demande aussi de prendre en considération leurs attitudes envers les langues apprises/enseignées ou, en d'autres termes, les représentations sociales selon lesquelles les apprenants fonctionnent en classe : « On entend par représentation sociale, avec la psycho-sociologie, une connaissance spontanée, socialement élaborée et partagée relativement à un objet. Cette notion est souvent rapprochée d'autres comme : stéréotype, cliché ou préjugé. Ces représentations collectives ne sont pas nécessairement fondées, mais elles influencent les motivations à l'apprentissage" (Byram \& Beacco, 2003, p.42-43). En effet, les représentations relatives à la langue peuvent concerner différents aspects qui conditionnent, ne serait-ce que partiellement, le travail en classe de langue. Il est notamment question de :

- la difficulté que l'apprentissage de la langue suppose : « on peut par exemple surestimer les difficultés de prononciation ou celles que présentent les systèmes d'écriture de ces langues » (ibid., p. 43);

- la beauté de la langue, qui fait penser non seulement à la phonétique et à la prosodie, mais aussi au prestige des œuvres littéraires et musicales ;

- l'utilité de la langue ou, en d'autres termes, le profit que l'on peut en tirer soit dans la vie professionnelle, soit dans la vie privée ;

- la valeur éducative de la langue : « certaines langues sont tenues pour faciles; elles peuvent alors être suspectes, car leur apprentissage est considéré comme n'exigeant pas suffisamment d'efforts. D'autres peuvent être privilégiées, car elles favoriseraient les capacités (le raisonnement, en particulier) » (ibid.).

Dans le contexte d'apprentissage de la LE2, il semble que non seulement l'utilité de cette langue mais aussi sa difficulté supposée soient des facteurs conditionnant son apprentissage efficace. En effet, la difficulté supposée de la langue renvoie tout de suite à deux notions : distance topologique et psychotopologique. La distance topologique peut être définie en tant que degré de parenté formel (génétique) entre les langues ou les 
familles de langues. La distance en question est mesurée à l'aide des traits communs entre les langues, par suite de comparaisons de nature purement linguistique (Chłopek, 2011, p.155). Par voie de conséquence, on distingue les langues: apparentées, voisines et éloignées. Les langues apparentées, ce sont des langues qui appartiennent à la même famille (langue romanes, germaniques, slaves, etc.) : «leur parenté génétique assure un maximum de similitudes linguistiques à tout niveau : phonologique, alphabétique, lexical, morphologique et syntaxique » (Caddéo \& Jamet, 2013, p. 51). Ainsi, l'apprentissage d'une langue qui appartient à la même famille qu'une autre connue par l'individu se fait plus facilement et, par conséquence, plus vite car «le temps d'accommodation à ce qui relève de la nouveauté se réduit considérablement et l'accès à la langue inconnue est facilité. Les conditions sont réunies pour permettre les transferts du savoir-faire et l'apprenant est encouragé à trouver les ressemblances plutôt que les différences; ce qui fait également évoluer les représentations sur les langues » (ibid.). S'il s'agit des langues voisines, ce sont des langues qui ont des liens communs par suite du contact qui résulte de raisons soit historiques, soit géographiques. Par exemple, le français et l'anglais peuvent être considérés comme des langues voisines: "Le français est [...] rapproché de l'anglais; cette langue, au Moyen-Âge a en effet beaucoup emprunté de mots au français du fait de la présence à la Cour d'Angleterre » (ibid., p. 52). Viennent ensuite les langues éloignées, à savoir les langues qui ne sont ni apparentées, ni voisines, mais qui sont bien différentes. La question qui se pose est celle de savoir s'il existe un certain nombre de régularités entre ces langues qui pourraient faciliter leur apprentissage. Or, comme l'énoncent $\mathrm{S}$. Caddéo \& M.-C. Jamet (ibid.), «il est possible aussi de comprendre des langues fort différentes - ni proches ni voisines - [...] en s'appuyant sur tout ce que l'on sait déjà (expérience du monde, le fait de connaître au moins une langue - la sienne - ou bien d'en connaître plusieurs), et être en mesure de réaliser une tâche donnée, à condition qu'elle soit bien guidée ». Travailler les langues éloignées débouche sur la question de la distance dite psychotopologique, à savoir la distance entre les langues qui est perçue, de façon subjective, par les apprenants/usagers des langues (Chłopek, 2011, p. 158). Or, la façon dont ils perçoivent la distance en question a un impact sur l'efficacité de l'enseignement/ apprentissage, car plus cette distance est raccourcie, plus le transfert entre les savoirs langagier et linguistique est efficace. Il faut aussi remarquer que la distance psychotopologique ne doit pas forcément concerner tout le système linguistique, mais seulement un domaine particulier : un sous-système linguistique, un champ thématique, etc. De plus, la distance psychotopologique a un caractère évolutif : elle change avec l'âge de l'individu, avec le niveau du développement cognitif ou encore avec le niveau du développement de la conscience métacognitive que nous avons brièvement décrite en début d'article. Il est intéressant de voir qu'au commencement de l'apprentissage, les élèves perçoivent avant tout les ressemblances formelles entre les unités linguistiques de base ; la réflexion sur les ressemblances sémantiques et/ou fonctionnelles vient plus tard. Il en résulte que les élèves débutants se concentrent avant tout sur les formes linguistiques, alors que ceux plus avancés dans leur apprentissage focalisent plutôt leur attention sur les aspects sémantiques de la langue qu'ils sont en train d'apprendre, de même que sur les ressemblances dans les mécanismes de production. Enfin, la question est de savoir si la capacité à percevoir la distance psychotopologique est une capacité consciente ou inconsciente. En effet, les avis des chercheurs sont partagés (Chłopek, 201, p. 162). D'un côté, il s'agit d'un processus latent, car les ressemblances entre les langues semblent être tout à fait naturelles aux apprenants (par exemple, certaines formes linguistiques entre l'allemand et le hollandais). Mais, de l'autre côté, le fait qu'il y ait une 
relation entre le développement de la conscience métalinguistique et la psychotopologie suggère qu'il s'agit d'un processus conscient.

En résumé, le transfert des savoirs et savoir-faire est presque toujours conditionné par la représentation sociale que l'apprenant a sur la langue, avant tout sur la distance qui se met en place entre les langues qu'il connait et la langue qu'il est en train d'apprendre. Qu'il s'agisse de la distance objective ou subjective, l'enseignement/apprentissage d'une langue est toujours situé dans un contexte et ce n'est qu'en le prenant en considération que l'enseignant de langue étrangère peut efficacement développer la compétence plurilingue des apprenants en favorisant le transfert positif entre les langues qui font partie du répertoire plurilingue de l'apprenant. Les questions qui doivent se mettre en place peuvent-être formulées de la façon suivante :

- Quelle est le lien de parenté entre la langue maternelle des apprenants et la langue cible?

- Quelle est le degré de parenté entre les autres langues étrangères de l'apprenant et la langue cible?

- Quelles sont les représentations des apprenants concernant la langue cible? La trouvent-ils utile? Difficile? Belle? Sophistiquée ? etc.

- Quel est le degré de leur savoir sur la langue en tant que système où l'on retrouve des régularités?

- Quel est le degré de leur conscience métalinguistique? Savent-ils en profiter pour apprendre la langue cible?

C'est autour de ces questions que devrait s'organiser le travail de l'enseignant qui voudrait puiser dans le répertoire langagier de l'apprenant lors de l'enseignement/ apprentissage d'une langue étrangère. Cette perspective semble être cruciale en classe de deuxième langue étrangère (il est d'habitude question d'une autre langue que l'anglais). En effet, l'anglais est la langue qui domine les classes de langue d'aujourd'hui. Même si cette hégémonie se voit justifiée, car l'anglais "permet une économie d'échelle dans les échanges, désormais étendus à l'ensemble du monde » (Byram \& Beacco, 2003, p. 27), elle met en danger d'autres langues qui sont souvent perçues par les élèves comme plus difficiles et avant tout moins utiles, ce qui a un impact négatif sur l'apprentissage. Ainsi, pour motiver les apprenants, l'enseignant devrait montrer aux élèves comment puiser dans leur répertoire langagier afin de profiter de la synergie qui se met en place entre les langues qui en font partie.

Dans la suite, nous montrerons les résultats d'une étude empirique que nous avons menée auprès de collégiens polonais (17 ans) qui apprennent le français comme la LE2 (après l'anglais). L'objectif de l'étude était de voir si les élèves avaient atteint le niveau imposé par le Programme national - A2 en l'occurrence -, et s'ils savaient recourir, lors de l'apprentissage du français, aux stratégies résultant de leur répertoire langagier.

\section{3. Étude empirique}

\subsection{Le contexte de l'étude}

11 L'étude empirique dont nous présenterons les résultats ci-dessous a été menée auprès de collégiens polonais apprenant le français en tant que deuxième langue étrangère. Il faut 
préciser que la Pologne peut être qualifiée de pays monolingue pour les raisons suivantes (Wojciechowska, 2010, p. 67):

- en Pologne, il n'existe qu'une langue officielle - le polonais - qui est utilisée par plus de $95 \%$ des habitants du pays ;

- la majorité des Polonais (plus de $95 \%$ ) définissent leur nationalité en tant que nationalité polonaise ;

- en Pologne, le nombre d'immigrés est relativement bas de même que le nombre de minorités nationales.

Il en résulte que le contact avec les langues étrangères a lieu, avant tout, à l'école, car les apprenants ont peu de possibilités d'utiliser les langues étrangères dans des situations de communication informelles, notamment s'il s'agit de la deuxième langue étrangère (Szpotowicz et al., 2013). Or, depuis l'année scolaire 2009-2010 ou l'année de l'introduction du nouveau Programme national, une langue étrangère est obligatoire dès le début de l'école primaire alors que, au collège et au lycée, les élèves apprennent obligatoirement deux langues étrangères, ce qui veut dire qu'au cours de la scolarisation obligatoire, les apprenants peuvent étudier jusqu'à trois langues étrangères, ce que montre le schéma ci-dessous.

Tableau 1. L'enseignement des langues étrangères en Pologne

\begin{tabular}{|l|l|l|}
\hline l'école primaire & le collège & le lycée \\
\hline LE1 & LE2 & \\
\hline & \multicolumn{3}{l}{} \\
\hline ou & LE2 & LE3 \\
\hline L1 & \multicolumn{2}{l|}{} \\
\hline \multicolumn{3}{|l}{} \\
\hline ou & LE2 & LE3 \\
\hline LE1 &
\end{tabular}

13 Il faut aussi noter que les objectifs d'enseignement/apprentissage des langues étrangères renvoient aux composantes de la compétence plurilingue. En effet, à chaque étape éducative (école primaire et secondaire), l'élève est amené non seulement à recourir à des stratégies communicatives et compensatoires lors de la communication dans la langue cible, mais il doit aussi posséder la conscience linguistique qui lui permettrait, selon les concepteurs du Programme national, de percevoir, entre autres, les ressemblances et différences entre les langues. On peut donc supposer que l'organisation de l'enseignement/apprentissage des langues permet de développer la compétence plurilingue des apprenants en les faisant réfléchir sur les langues qui composent leur répertoire langagier pour pouvoir profiter de la synergie qui en découle afin de renforcer 
les savoir-faire langagiers, notamment dans la deuxième langue étrangère dont l'apprentissage commence plus tard (au niveau du collège).

\subsection{Le groupe cible, les objectifs et les hypothèses de la recherche} apprenant le français en tant que LE2 (après l'anglais) depuis 3 ans (dernière classe du collège) dès le niveau débutant. Ils viennent de 4 écoles : 2 écoles sont situées dans la ville de Varsovie, 1 se trouve dans la banlieue de Varsovie et 1 est localisée à l'Est de la Pologne. Notre étude a pour objectifs de/d' :

- constater si les élèves ont atteint le niveau A2 en français, à savoir le niveau imposé par le Programme national dans les compétences réceptives (compréhensions : orale et écrite);

- examiner les stratégies auxquelles recourent les élèves lors de l'écoute et la lecture en langues étrangères ;

- vérifier si les apprenants puisent dans leur répertoire langagier lors de l'écoute et de la lecture en français.

Les hypothèses que nous avons formulées pour les besoins de la présente recherche découlent des objectifs présentés ci-dessus et se présentent de la façon suivante :

- les élèves n'ont pas atteint le niveau désiré en compétences réceptives en français, le niveau A2 en l'occurrence ;

- l'éventail des stratégies d'écoute et de lecture reste modeste;

- les élèves ne puisent pas dans leur répertoire langagier lors de l'écoute et de la lecture en français.

En formulant nos hypothèses, nous nous sommes appuyé sur notre expérience en tant que professeur de FLE au lycée. En effet, les apprenants qui arrivent à l'école n'ont pas l'habitude de puiser dans la synergie qui devrait se mettre en place entre les langues qu'ils connaissent. Au contraire, ils ont plutôt tendance à percevoir les langues qu'ils apprennent de façon cloisonnée, ce qui veut dire qu'ils ne recourent pas aux stratégies qui résulteraient de leur compétence plurilingue. Même situation pour le niveau de la langue atteint après trois ans d'apprentissage au collège. Rares sont les élèves qui présentent un vrai niveau A2 qui permettrait la continuité de l'apprentissage: leurs compétences langagières oscillent plutôt entre le niveau A1 et A1+ (Szpotowicz et al., 2013).

\subsection{Les outils}

Dans notre recherche, nous nous sommes servi de deux tests. Le premier visait la compréhension de l'oral au niveau A2, le second la compréhension des écrits, aussi au niveau A2. Les deux tests se composaient de trois activités fermées et vérifiaient les capacités suivantes: définir l'idée générale du texte, définir le contexte de l'enregistrement et trouver des informations précises; en un mot, les capacités qui constituent les objectifs de l'enseignement/apprentissage des langues au collège. Dans la recherche, nous nous sommes aussi servi de quatre « questionnaires stratégiques » dont les objectifs sont présentés dans le tableau ci-dessous. 
Tableau 2. Objectifs des questionnaires stratégiques

\begin{tabular}{|l|l|}
\hline $\begin{array}{l}\text { Numéro } \\
\text { questionnaire }\end{array}$ & Objectif \\
\hline 1 & $\begin{array}{l}\text { examiner l'éventail des stratégies relatives à la compréhension de } \\
\text { l'oral dans les LE }\end{array}$ \\
\hline 2 & $\begin{array}{l}\text { examiner si les élèves utilisent leur répertoire langagier lors de } \\
\text { l'écoute en français }\end{array}$ \\
\hline 3 & $\begin{array}{l}\text { examiner l'éventail des stratégies relatives à la compréhension des } \\
\text { écrits dans les LE }\end{array}$ \\
\hline 4 & $\begin{array}{l}\text { examiner si les élèves utilisent leur répertoire langagier lors de la } \\
\text { lecture en français }\end{array}$ \\
\hline
\end{tabular}

Dans chacun des questionnaires, les élèves ont dû choisir un chiffre entre 1 et 5 . Les valeurs attribuées aux chiffres sont les suivants : $1=$ jamais ; $2=$ rarement ; $3=$ parfois ; 4 = souvent, 5 = toujours.

\subsection{La présentation des résultats}

\section{L'analyse des résultats des tests}

19 Le tableau suivant montre l'analyse des résultats des élèves, obtenus lors du test 1 (compréhension de l'oral) et du test 2 (compréhension des écrits).

Tableau 3. Analyse des tests - compréhension orale ( $\mathrm{T} 1$ ) et compréhension écrite (T2)

\begin{tabular}{|l|l|l|l|l|l|}
\hline $\begin{array}{l}\text { Numéro du } \\
\text { test }\end{array}$ & $\begin{array}{l}\text { Total à } \\
\text { obtenir }\end{array}$ & $\begin{array}{l}\text { Résultat } \\
\text { moyen }\end{array}$ & $\begin{array}{l}\text { Écart } \\
\text { type }\end{array}$ & $\begin{array}{l}\text { Indice de } \\
\text { facilité }\end{array}$ & $\begin{array}{l}\text { Interprétation de l'indice } \\
\text { de facilité }\end{array}$ \\
\hline T1 & 15 & 9,5 & 3,05 & $0,63(3)$ & moyennement difficile \\
\hline T2 & 16 & 11,78 & 3,31 & 0,73 & facile \\
\hline
\end{tabular}

20 Il en résulte que le test visant la compréhension de l'oral était légèrement plus facile que le test de la compréhension des écrits. Néanmoins, l'écart type est relativement élevé, ce qui prouve que la dispersion des résultats est grande et, par voie de conséquence, nous ne pouvons pas généraliser nos conclusions. Il est aussi intéressant de voir l'étalage des résultats obtenus par les élèves que nous présentons ci-dessous. 
Figure 1. Résultats individuels - compréhension orale

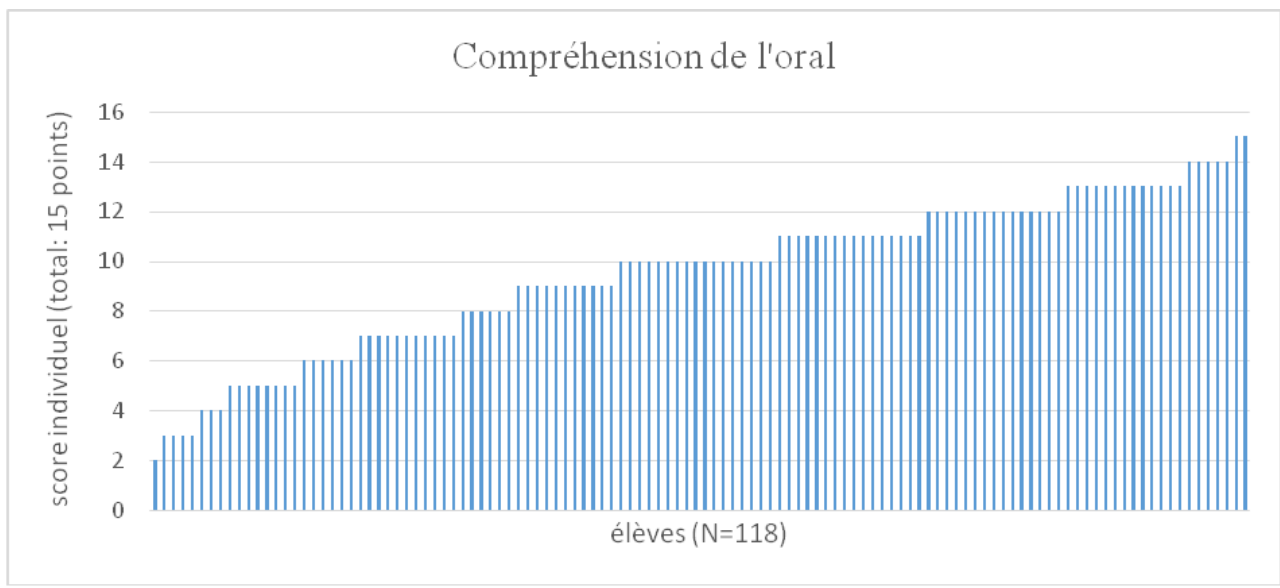

Figure 2. Résultats individuels - compréhension écrite

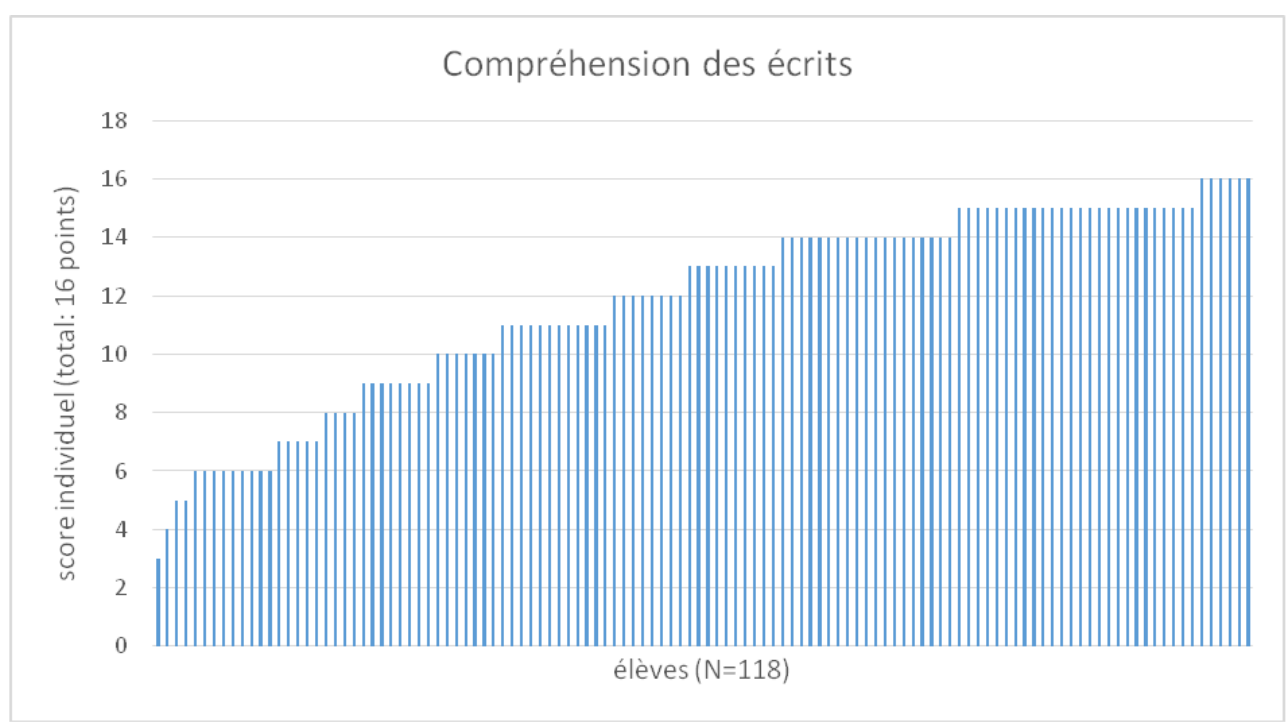

En admettant que $50 \%$ du total permettent d'atteindre le niveau désiré (A2 dans le cas de notre étude), on constate qu'il y a quand même un nombre assez élevé d'apprenants qui n'ont pas atteint ce niveau ou bien qui l'ont à peine atteint, notamment pour la compréhension de l'oral qui passe pour une compétence plus difficile en classe de langue.

\section{L'analyse des « questionnaires stratégiques »}

Quant à l'analyse des réponses concernant les stratégies d'écoute et de lecture, elle est présentée dans les tableaux qui suivent.

\section{Écoute en LE}


Tableau 4. Stratégies de l'écoute en langue étrangère

\begin{tabular}{|c|c|c|c|}
\hline & Avant l'écoute & Moyenne & $\begin{array}{l}\text { Écart } \\
\text { type }\end{array}$ \\
\hline 1. & $\begin{array}{l}\text { Lire attentivement les consignes pour comprendre les tâches à } \\
\text { réaliser. }\end{array}$ & 4,35 & 0,93 \\
\hline 2. & $\begin{array}{l}\text { Se rappeler les textes qui portaient sur un pareil sujet et les mots qui } \\
\text { concernent la thématique du texte. }\end{array}$ & 2,80 & 1,21 \\
\hline \multirow[t]{2}{*}{3.} & Souligner les mots clés dans la consigne. & 2,43 & 1,3 \\
\hline & Pendant l'écoute & & \\
\hline 4. & $\begin{array}{l}\text { Saisir l'idée générale du texte pendant la première écoute (Quel est le } \\
\text { type de texte? Qui parle ? À propos de quoi ? Quand ? pourquoi ?) }\end{array}$ & 4,19 & 0,97 \\
\hline 5. & $\begin{array}{l}\text { Essayer de comprendre les mots inconnus qui sont nécessaires à la } \\
\text { compréhension du texte (contexte phrastique par exemple) }\end{array}$ & 4,06 & 0,99 \\
\hline 6. & $\begin{array}{l}\text { Ne pas se décourager par la méconnaissance des mots inconnus en les } \\
\text { remplaçant par truc machin }\end{array}$ & 3,86 & 1,22 \\
\hline 7. & $\begin{array}{l}\text { Se référer à son expérience et à son savoir déclaratif pour prévoir le } \\
\text { contenu du texte. }\end{array}$ & 3,46 & 1,17 \\
\hline 8. & Définir les rapports entre les différentes parties du texte. & 3,22 & 1,17 \\
\hline 9. & $\begin{array}{l}\text { Focaliser l'attention sur l'intonation des interlocuteurs afin de définir } \\
\text { leurs émotions pour mieux comprendre le texte. }\end{array}$ & 3,53 & 1,20 \\
\hline 10. & $\begin{array}{l}\text { Accorder de l'importance à la grammaire (temps et modes pour situer } \\
\text { le texte dans le temps, terminaisons grammaticales pour définir les } \\
\text { sujets parlants) }\end{array}$ & 3,28 & 1,38 \\
\hline \multirow[t]{2}{*}{11.} & Ne pas se décourager. & 3,71 & 1,35 \\
\hline & Après l'écoute & & \\
\hline 12. & $\begin{array}{l}\text { Réfléchir sur d'autres stratégies qui peuvent être mises en œuvre lors } \\
\text { de l'écoute. }\end{array}$ & 2,61 & 1,11 \\
\hline
\end{tabular}

\section{Écoute en FLE}

Tableau 5. Stratégies de l'écoute en français langue étrangère

\begin{tabular}{|l|l|l|l|}
\hline Pendant l'écoute & Moyenne & $\begin{array}{l}\text { Écart } \\
\text { type }\end{array}$ \\
\hline
\end{tabular}




\begin{tabular}{|l|l|l|l|}
\hline 1. & $\begin{array}{l}\text { Être conscient que les conversations en français ressemblent aux } \\
\text { conversations en polonais, en anglais ou dans d'autres langues. }\end{array}$ & $\mathbf{2 , 9 3}$ & $\mathbf{1 , 3 0}$ \\
\hline 2. & $\begin{array}{l}\text { Lire attentivement la consigne en focalisant son attention sur les mots } \\
\text { qui ressemblent à des mots connus en polonais, en anglais ou dans }\end{array}$ & $\mathbf{3 , 9 2}$ & $\mathbf{1 , 0 4}$ \\
\hline 3. & $\begin{array}{l}\text { Définir le genre du texte et se rappeler les genres pareils en polonais, } \\
\text { en anglais ou dans d'autres langues. }\end{array}$ & $\mathbf{3 , 2 9}$ & $\mathbf{1 , 2 1}$ \\
\hline 4. & $\begin{array}{l}\text { Se demander si les mots inconnus en français ressemblent à des mots } \\
\text { connus en polonais, en anglais ou dans une autre langue. }\end{array}$ & $\mathbf{3 , 8 9}$ & $\mathbf{1 , 1 2}$ \\
\hline 5. & $\begin{array}{l}\text { Remplacer les mots inconnus par des mots connus en polonais, en } \\
\text { anglais ou dans une autre langue. }\end{array}$ & $\mathbf{3 , 7 5}$ & $\mathbf{1 , 1 7}$ \\
\hline 6. & $\begin{array}{l}\text { Focaliser son attention sur les mots connus ou mots qui ressemblent à } \\
\text { des mots connus en polonais, en anglais ou dans une autre langue. } \\
\text { autre langue est utile lors de la compréhension du texte en français. }\end{array}$ & $\mathbf{2 , 2 3}$ & $\mathbf{1 , 3 4}$ \\
\hline 7. & $\begin{array}{l}\text { Saisir les mots qui expriment les émotions et qui ressemblent à des } \\
\text { mots connus en polonais, anglais ou dans une autre langue. }\end{array}$ & $\mathbf{3 , 2 4}$ & $\mathbf{1 , 2 9}$ \\
\hline Après l'écoute & $\mathbf{1 , 2 2}$ \\
\hline connaissance du polonais, de l'anglais ou d'une autre langue. & $\mathbf{1 , 2 1}$ \\
\hline
\end{tabular}

L'analyse des résultats montre que les élèves disposent d'un éventail assez riche de stratégies qu'ils activent lors de l'écoute en langues étrangères. Néanmoins, il leur manque des stratégies qui demandent de la réflexion soit avant, soit après l'écoute. Il faut aussi remarquer que l'écart type est relativement grand, ce qui ne nous permet pas, encore une fois, de généraliser nos conclusions. Quant aux stratégies qui sont liées au répertoire langagier des apprenants, elles sont relativement peu développées, ce qui prouve que la conscience métalinguistique des apprenants nécessite encore un travail intense. Il est à noter que l'écart type est lui aussi important, ce qui prouve la dispersion des résultats. L'analyse des données permet de constater qu'il existe une corrélation entre les résultats du test et l'activation - relativement pauvre - des stratégies d'écoute relevant du répertoire langagier des apprenants.

\section{Lecture en LE}

Tableau 6. Stratégies de la lecture en langue étrangère

\begin{tabular}{|l|l|l|l|}
\hline Avant la lecture & Moyenne & $\begin{array}{l}\text { Écart } \\
\text { type }\end{array}$ \\
\hline
\end{tabular}




\begin{tabular}{|c|c|c|c|}
\hline 1. & $\begin{array}{l}\text { Lire attentivement les consignes pour comprendre les tâches à } \\
\text { réaliser. }\end{array}$ & 4,06 & 1,01 \\
\hline \multirow[t]{2}{*}{2.} & $\begin{array}{l}\text { Se rappeler les textes qui portaient sur un pareil sujet et les mots qui } \\
\text { concernent la thématique du texte. }\end{array}$ & 3,04 & 1,32 \\
\hline & Pendant la lecture & & \\
\hline 3. & Lire le texte en entier pour saisir son idée générale. & 4,17 & 1,07 \\
\hline 4. & $\begin{array}{l}\text { Essayer de comprendre les mots inconnus qui sont nécessaires à la } \\
\text { compréhension du texte (contexte phrastique par exemple). }\end{array}$ & 4,08 & 1,20 \\
\hline 5. & $\begin{array}{l}\text { Je lis une phrase jusqu'à la fin. Ne pas se décourager par la } \\
\text { méconnaissance des mots inconnus en les remplaçant par truc machin }\end{array}$ & 4,06 & 1,13 \\
\hline 6. & $\begin{array}{l}\text { Se référer à son expérience et à son savoir déclaratif pour prévoir le } \\
\text { contenu du texte. }\end{array}$ & 3,64 & 1,17 \\
\hline 7. & Définir les rapports entre les différentes parties du texte. & 3,59 & 1,30 \\
\hline 8. & Focaliser l'attention sur la ponctuation présente dans le texte. & 3,69 & 1,23 \\
\hline 9. & $\begin{array}{l}\text { Accorder de l'importance à la grammaire (temps et modes pour situer } \\
\text { le texte dans le temps, terminaisons grammaticales pour définir les } \\
\text { sujets parlants). }\end{array}$ & 3,67 & 1,25 \\
\hline \multirow[t]{2}{*}{10.} & Ne pas se décourager. & 3,64 & 1,54 \\
\hline & Après la lecture & & \\
\hline 11. & $\begin{array}{l}\text { Relire le texte ou des fragments pour vérifier si on l'/les a bien } \\
\text { compris. }\end{array}$ & 4,07 & 1,16 \\
\hline 12. & $\begin{array}{l}\text { Réfléchir sur les stratégies mises en œuvre lors de la lecture et évaluer } \\
\text { leur utilité. }\end{array}$ & 2,78 & 1,33 \\
\hline 13. & $\begin{array}{l}\text { Réfléchir sur d'autres stratégies qui peuvent être mises en œuvre lors } \\
\text { de l'écoute. }\end{array}$ & 2,79 & 1,29 \\
\hline
\end{tabular}

\section{Lecture en FLE}

Tableau 7. Stratégies de la lecture en français langue étrangère

\begin{tabular}{|l|l|l|l|}
\hline Pendant la lecture & Moyenne & $\begin{array}{l}\text { Écart } \\
\text { type }\end{array}$ \\
\hline $\mathbf{1 .}$ & $\begin{array}{l}\text { Observer attentivement tous les éléments qui accompagnent le texte } \\
\text { pour voir s'il y a des éléments qui ressemblent à des éléments que je } \\
\text { connais dans d'autres langues. }\end{array}$ & $\mathbf{4 , 0 2}$ & $\mathbf{1 , 1 6}$ \\
\hline
\end{tabular}




\begin{tabular}{|c|c|c|c|}
\hline 2. & $\begin{array}{l}\text { Définir le genre du texte et se rappeler les genres pareils en polonais, } \\
\text { en anglais ou dans d'autres langues. }\end{array}$ & 3,56 & 1,23 \\
\hline 3. & $\begin{array}{l}\text { Lire le texte en entier pour saisir son idée principale en focalisant son } \\
\text { attention sur les mots que l'on connaît ou qui ressemblent à des mots } \\
\text { connus dans d'autres langues. }\end{array}$ & 3,86 & 1,10 \\
\hline 4. & $\begin{array}{l}\text { Se demander si les mots inconnus en français ressemblent à des mots } \\
\text { connus en polonais, en anglais ou dans une autre langue. }\end{array}$ & 3,75 & 1,20 \\
\hline 5. & $\begin{array}{l}\text { Remplacer les mots inconnus par des mots connus en polonais, en } \\
\text { anglais ou dans une autre langue. }\end{array}$ & 3,64 & 1,28 \\
\hline 6. & $\begin{array}{l}\text { Lire les mots inconnus à voix haute et se demander s'ils ne } \\
\text { ressemblent pas à des mots connus dans d'autres langues. }\end{array}$ & 2,73 & 1,44 \\
\hline 7. & $\begin{array}{l}\text { Analyser les constructions lexicales inconnues et se demander si de } \\
\text { pareilles structures existent en polonais, en anglais ou dans une autre } \\
\text { langue connue. }\end{array}$ & 2,93 & 1,29 \\
\hline 8. & $\begin{array}{l}\text { Observer les constructions lexicales dont une partie est inconnue pour } \\
\text { voir si de pareilles structures existent en polonais, en anglais ou dans } \\
\text { une autre langue connue. }\end{array}$ & 3,05 & 1,31 \\
\hline 9. & $\begin{array}{l}\text { Analyser les constructions grammaticales inconnues et se demander si } \\
\text { de pareilles structures existent en polonais, en anglais ou dans une } \\
\text { autre langue connue. }\end{array}$ & 3,06 & 1,19 \\
\hline 10. & $\begin{array}{l}\text { Relire le texte pour voir si les hypothèses sur les éléments inconnus } \\
\text { ont été correctes. }\end{array}$ & 3,53 & 1,32 \\
\hline & Après la lecture & & \\
\hline 11. & $\begin{array}{l}\text { Réfléchir à quel point la connaissance du polonais, de l'anglais ou } \\
\text { d'une autre langue est utile lors de la compréhension du texte en } \\
\text { français. }\end{array}$ & 2,70 & 1,32 \\
\hline 12. & $\begin{array}{l}\text { Réfléchir sur d'autres stratégies de lecture en FLE qui mettent en } \\
\text { œuvre la connaissance du polonais, de l'anglais ou d'une autre langue. }\end{array}$ & 2,54 & 1,32 \\
\hline
\end{tabular}

En analysant les questionnaires concernant les stratégies mise en œuvre lors de la lecture, nous constatons que ces stratégies sont plus présentes dans l'esprit des élèves que les stratégies concernant l'écoute. Néanmoins, les stratégies résultant du répertoire langagier des apprenants sont aussi pauvres que dans le cas des mêmes stratégies concernant l'écoute. Ce qui veut probablement dire que la conscience métalinguistique des apprenants n'est pas encore suffisamment développée et demande du travail. Dans les deux groupes de stratégies, l'écart type est élevé, ce qui signifie que les conclusions que nous en tirons sont loin d'être représentatives. Comme pour l'analyse des données concernant l'écoute en FLE, nous osons constater que les résultats de la lecture en FLE pourraient être meilleurs, si les élèves recouraient à leur répertoire plurilingue. 


\subsection{Les conclusions} exhaustive, montrent que même si la notion de la compétence plurilingue s'est déjà ancrée bel et bien dans la théorie didactique, il lui faut encore du temps pour exister vraiment dans les pratiques de classe, ce que nous avons essayé de prouver lors de notre recherche. Alors, amener l'apprenant à une réflexion consciente sur la langue lui permettra de développer la compétence plurilingue qui conditionne non seulement l'apprentissage des langues en général, mais avant tout l'apprentissage de la LE2 qui reste souvent à l'ombre de la L1, l'anglais en l'occurrence. Pour ce faire, l'enseignant doit éveiller chez ses élèves la conscience métalinguistique en passant par le processus de conscientisation qui « est une démarche active permettant le plus souvent de modifier à la fois ses représentations et son attitude » (Caddéo \& Jamet, 2013, p. 61). L'apprenant à conscience métalinguistique développée sait :

- identifier ses savoirs et savoir-faire dans le domaine de l'apprentissage des langues;

- les transférer pour l'apprentissage de nouvelles langues («par exemple envisager la maîtrise des stratégies de lecture en langue étrangère comme une compétence généralisable sur plusieurs langues », ibid.);

- évaluer consciemment ses représentations sur les langues : sentiment de distance entre les langues qu'il apprend, sur ce qu'« apprendre » ou « connaitre » une langue veut dire.

C'est ce processus de conscientisation qui constitue un nouveau défi pour la didactique des langues étrangères d'aujourd'hui, notamment pour la didactique des langues étrangères autres que l'anglais.

\section{BIBLIOGRAPHIE}

ARDITTY, J. (2008). « Conscience linguistique, conscience langagière ». In : Candelier, M. et al. (dir.), Conscience du plurilinguisme. Pratiques, représentations et interventions. Rennes : Presses universitaires de Rennes, p. 35-49.

Guide pour l'élaboration des politiques linguistiques éducatives en Europe. De la diversité linguistique à l'éducation plurilingue. Strasbourg : Conseil de l'Europe.

BOURGUignon, C. \& CANDELIER, M. (éds) (2014). « La place de la langue maternelle dans la construction par l'élève des notions grammaticales pour l'apprentissage requises pour l'apprentissage d'une langue étrangère ». In : Troncy, C. et al. (dirs), Didactique du plurilinguisme. approches plurielles des langues et des cultures. Autour de Michel Candelier. Rennes : Presses universitaires de Rennes, p. 89-107.

CADDÉO, S. \& JAMET, M.-C. (éds) (2013). L'intercompréhension : une autre approche pour l'enseignement des langues. Paris : Hachette.

CHŁOPEK, Z. (éd.) (2011). Nabywanie jĘzyków trzecich i kolejnych oraz wielojĘzyczność. Aspekty psycholingwistyczne (i inne). Wrocław : Wydawnictwo Uniwersytetu Wrocławskiego. 
HUVER, E. \& SPRINGER, C. (éds) (2011). L'évaluation en langues. Nouveaux enjeux et perspectives. Paris : Didier.

PAIKEDAY, T. M. (éd.) (2003). The Native Speaker Is Dead! Tokyo : Lexicography.

ROBERT, J.-P. \& ROSEN, É. (éds) (2010). Dictionnaire pratique du CECR. Paris : Ophrys.

SZPoтоwICZ, M. et al. (éds) (2013). Europejskie Badanie Kompetencji JĘzykowych ESLC. Raport krajowy 2011. Warszawa : Instytut Badań Edukacyjnych.

WOJCIECHOWSKA, B. (2010). « WielojĘzyczność w polskim kontekście edukacyjnym ». Neofilolog 35, p. 63-74.

\section{Documents officiels}

BEACCO, J.-C. \& BYRAM, M. (éds) (2003). Guide pour l'élaboration des politiques linguistiques éducatives en Europe. Strasbourg : Conseil de l'Europe. En ligne : http://www.coe.int/t/dg4/linguistic/

Guide_niveau3_FR.asp.

Cadre européen commun de référence pour les langues : apprendre, enseigner, évaluer (2001).

Strasbourg : Conseil de l'Europe. En ligne : http://www.coe.int/t/dg4/linguistic/cadre1_fr.asp.

Conclusions du Conseil du 12 mai 2009 concernant un cadre stratégique pour la coopération européenne dans le domaine de l'éducation et de la formation (2009). Journal officiel de l'Union européenne C 119/2. En ligne : http://eur-lex.europa.eu/legal-content/FR/TXT/HTML/?uri=CELEX:52009XG0528 (01)\&from=FR (consulté le 10/10/2015).

Recommandation du Parlement européen et du Conseil du 18 décembre 2006 sur les compétences clés pour l'éducation et la formation tout au long de la vie (2006). Journal officiel de l'Union européenne L 394/10. En ligne : http://eur-lex.europa.eu/legal-content/FR/TXT/HTML/? uri=CELEX:32006H0962\&from=FR (consulté le 10/10/2015).

Résolution du Conseil du 21 novembre 2008 relative à une stratégie européenne en faveur du multilinguisme (2008). Journal officiel de l'Union européenne C 320/1. En ligne : http://eur-lex.europa.eu/legalcontent/FR/TXT/HTML/?uri=CELEX:32008G1216(01)\&from=FR (consulté le 10/10/2015).

\section{NOTES}

1. that native speakers are made, not born, but you are in fact including many different levels of dialects, educations, awareness, etc. in this overall concept. Just to give what might prove to be a helpful angle or two. There are people who are super-conscious of language and langue use - poets, writers, punsters, people who are making up new words either for fun or for living; and they are people who are, generally speaking, unconscious of their language use or that of other people (although they may be able to characterise someone else's speech as "hick" or even imitate it, without being able to label or identify elements which lead to that characterisations). 


\section{RÉSUMÉS}

Le présent article a pour objectif d'analyser comment on peut développer la compétence plurilingue en classe pour la deuxième langue étrangère (LE2) dans un pays monolingue. Les apports théoriques seront illustrés par l'analyse de résultats d'une recherche menée auprès d'élèves polonophones (âgés d'entre 15 et 16 ans) apprenant le français en tant que LE2 (après l'anglais).

This article aims to analyse how we can develop the multilingual competence in a second foreign language class (FL2) in a monolingual country. The theoretical contributions will be illustrated by the analysis of the results of a research conducted among Polish speakers pupils (aged between 15 and 16 years) learning French as a FL2 (after English).

\section{INDEX}

Mots-clés : plurilinguisme, compétence plurilingue, écoute, lecture, FLE, élèves polonais

Keywords : multilingualism, multilingual competence, listening, reading, FFL, Polish students

\section{AUTEUR}

\section{RADOSŁAW KUCHARCZYK}

